\title{
Generation of Length Distribution, Length Diagram, Fibrogram, and Statistical Characteristics by Weight of Cotton Blends
}

\author{
B. Azzouz, M. Ben Hassen, and F. Sakli
}

Received 1 August 2007; Accepted 12 December 2007

Recommended by F. Gao

\begin{abstract}
The textile fibre mixture as a multicomponent blend of variable fibres imposes regarding the proper method to predict the characteristics of the final blend. The length diagram and the fibrogram of cotton are generated. Then the length distribution, the length diagram, and the fibrogram of a blend of different categories of cotton are determined. The length distributions by weight of five different categories of cotton (Egyptian, USA (Pima), Brazilian, USA (Upland), and Uzbekistani) are measured by AFIS. From these distributions, the length distribution, the length diagram, and the fibrogram by weight of four binary blends are expressed. The length parameters of these cotton blends are calculated and their variations are plotted against the mass fraction $x$ of one component in the blend. These calculated parameters are compared to those of real blends. Finally, the selection of the optimal blends using the linear programming method, based on the hypothesis that the cotton blend parameters vary linearly in function of the components rations, is proved insufficient.
\end{abstract}

Copyright $\odot 2007$ B. Azzouz et al. This is an open access article distributed under the Creative Commons Attribution License, which permits unrestricted use, distribution, and reproduction in any medium, provided the original work is properly cited.

\section{INTRODUCTION}

Fibre length is a very important physical measure in cotton spinning industry. In common with most of cotton properties, it varies greatly between varieties and within the same variety due to growth environment. Length is related to other cotton fibre characteristics. Longer fibres are generally more uniform, finer, and stronger than shorter ones. Cotton length affects many parameters during the spinning process such as production efficiency, amount of waste, and cleaning degree. Yarn quality parameters such as strength, elongation, hairiness, and evenness are strongly correlated to the length of cotton fibres.

The fibre length of a cotton sample can only be fully described by its distribution, but fibre length distribution is an awkward way to compare cotton length. Therefore, certain characteristics (statistical parameters) of a fibre length distribution are often used to make comparison.

Several researches were interested in fibre-length analysis. Many of these researches studied the methods and the instruments measuring the fibre length.

Hertel [1], the inventor of the fibrograph, gives an optical method to plotting the fibrogram from a sample of parallel fibres. From this fibrogram, fibre length and fibre length uniformity of raw fibre samples can be determined by a geometric interpretation.
Landstreet [2] described the basic ideas of the fibrogram theory starting from a frequency diagram and establishing geometrical and probabilistic interpretations for single fibre length, two fibre length, and multiple fibre length populations.

Krowicki et al. [3, 4] applied a new approach to generate the fibrogram from the length array data similar to Landstreet method. They assumed a random catching and holding of fibres within each of the length groups generating a triangular distribution by relative weight for each length group.

Krowicki and Duckett [5] showed that the mean length and the proportion of fibres can be obtained from the fibrogram.

Zeidman et al. [6, 7] discussed the concept of short fibres content (SFC) and showed relationships between SFC and other fibre length parameters and functions. Later, they determined empirical relationships between SFC and the HVI (high volume instrument) length.

Other studies were conducted to generate fibre length parameters and to study the relationships between these parameters and their effects on the other fibre characteristics and on the end product quality $[8,9]$.

To produce yarn with acceptable quality and reasonable cost, it should be a blend of different varieties and categories of cotton. 
In literature, numerous studies were interested to optimise blends from different nature of fibre. However, few studies were dedicated to multicomponent cotton blends. These studies proved that an achievement of good quality and economic blend of different categories of cotton became more to more important.

Elmoghazy $[10,11]$ proposed a number of fibre selection techniques for a uniform multicomponent cotton blend and consistent output characteristics. Later, he studied sources of variability in a multicomponent cotton blend and critical factors affecting it.

Zeidman et al. [6] present equations necessary to determine the short fibre content (SFC) of a binary blend if the SFC and other fibre characteristics of each component are known.

Elmoghazy [12] used the linear programming method to optimise the cost of cotton fibre blends with respect to the quality criteria presented in linear equations. His work is interesting and deals with all cotton parameters, but it supposes that the blend characteristics and particularly length parameters are linear to the component ratios.

In this study, we expressed and studied the length distribution functions (the distribution $f(x)$, the length diagram $q(x)$, and the fibrogram $p(x))$ of cotton and of a multicomponent blend of cotton.

Then we studied the variation of length parameters in terms of the component ratios in the blend. To reach this object, we measure the biased-weight length distribution by Advanced Fibre Information System (AFIS) of each component of the blend. From these distributions, the length parameters of any blend (with any ratio) can be calculated. Thus their variations (particularly for binary blends) versus the ratios of the components can be known. Then the blend length parameters determined from the established blend distribution functions are compared to real blend parameters and good correlations are obtained.

The work presented in this paper is a part of number of works, in progress, that consist to use these length mathematical models with other nonlinear mathematical and statistical models, established to estimate the other cotton blend parameters (fineness, maturity, strength, and elongation), to optimise the selection of multicomponent cotton blends by using multiobjectives optimisation techniques.

\section{DEFINITIONS}

The fibre length can be described by its distribution by number that expresses the probability of occurrence $f_{n}(l)$ of a fibre within the length group $[l-d l, l+d l]$, or it can be described by its distribution by weight $f_{w}(l)$ that expresses the weight of fibres in each length group $[l-d l, l+d l]$.

In this study, we will be interested only in biased-weight length and $f_{w}(l)$ will be noted by $f(l)$.

A biased-weight diagram $q(l)$ can be obtained from the distribution by weight by summing $f(l)$ from the longest to the shortest length group defined by $[l-d l, l+d l]$;

$$
q(l)=\int_{l}^{\infty} f(t) d t
$$

Summing and normalising $q(l)$ from the longest length group to the shortest give the fibogram $p(l)$;

$$
p(l)=\frac{1}{\mathrm{ML}} \int_{l}^{\infty} q(t) d t
$$

where ML is the mean length by weight expressed in the following paragraph.

A family of parameters has been derived over the years. Mean length (ML), short fibre content (SFC\%), upper quartile length (UQL), upper half mean length (UHML), upper quartile mean length (UQML), span lengths (SL), uniformity index (UI\%), and uniformity ratio (UR\%) are the most length distribution parameters.

\subsection{Mean length by weight (ML)}

The mean length by weight ML is obtained by summing the product of fibre length and its weight, then dividing by the total weight of the fibres, which can be described by

$$
\mathrm{ML}=\int_{0}^{\infty} t f(t) d t
$$

\subsection{Variance of fibre length by weight (Var)}

The variance of fibre length by weight is obtained by summing the product of the square of the difference between fibre length and the mean length by weight and its weight, then dividing by the total weight of the fibres, which can be described by

$$
\operatorname{Var}=\int_{0}^{\infty}(t-\mathrm{ML})^{2} f(t) d t
$$

\subsection{Standard deviation of fibre length by weight $(\sigma)$}

The standard deviation of fibre length by weight $\sigma$ is the root square of the variance Var and it expresses the dispersion of fibres length:

$$
\sigma=\sqrt{\operatorname{Var}}
$$

\subsection{Coefficient of fibre length variation by weight (CV\%)}

The coefficient of variation of fibre length $\mathrm{CV} \%$ is the ratio of $\sigma$ divided by the mean length ML:

$$
\mathrm{CV} \%=\frac{\sigma}{\mathrm{ML}} \times 100
$$

\subsection{Upper quartile length by weight (UQL)}

The upper quartile length is defined as the length that is exceeded by $25 \%$ of fibres by weight:

$$
\int_{\mathrm{UQL}}^{\infty} f(t) d t=q(\mathrm{UQL})=0.25 \text {. }
$$




\subsection{Upper half mean length by weight (UHML)}

The UHML is the average length of the longest one-half of the fibres when they are divided on a weight basis:

$$
\mathrm{UHML}=\frac{1}{q(\mathrm{ME})} \int_{\mathrm{ME}}^{\infty} t f(t)=2 \int_{\mathrm{ME}}^{\infty} t f(t),
$$

where ME is the median length that exceeded by $50 \%$ of fibres by weight, then $q(\mathrm{ME})=0.5$.

\subsection{Upper quarter mean length by weight (UQML)}

The UQML is the average length of the longest one-quarter of the fibres when they are divided on a weight basis. So it is the mean length of the fibres longer than UQL:

$$
\mathrm{UQML}=\frac{1}{q(\mathrm{UQL})} \int_{\mathrm{UQL}}^{\infty} t f(t)=4 \int_{\mathrm{UQL}}^{\infty} t f(t) .
$$

\subsection{Span length by weight $\left(S L_{t \%}\right)$}

The percentage span length $t \%$ indicates the percentage of fibres that extends a specified distance or longer. The $2.5 \%$ and $50 \%$ are the most commonly used by industry. It can be calculated from the fibrogram as

$$
p\left(\mathrm{SL}_{t \%}\right)=\frac{t}{100}
$$

\subsection{Uniformity index (UI\%)}

UI\% is the ratio of the mean length divided by the upper half-mean length. It is a measure of the uniformity of fibre lengths in the sample expressed as a percent:

$$
\mathrm{UI} \%=\frac{\mathrm{ML}}{\mathrm{UHML}} \times 100 .
$$

\subsection{Uniformity ratio (UR\%)}

UR\% is the ratio of the $50 \%$ span length to the $2.5 \%$ span length. It is a smaller value than the UI\% by a factor close to 1.8:

$$
\mathrm{UR} \%=\frac{\mathrm{SL}_{50 \%}}{\mathrm{SL}_{2.5 \%}} \times 100 .
$$

\subsection{Short fibre content (SFC\%)}

SFC\% is the percentage by weight of fibres less than one half inch $(12.7 \mathrm{~mm})$. Mathematically, it is described as follows:

$$
\mathrm{SFC} \%=100 \times \int_{0}^{12.7} f(t) d t=100 \times(1-q(12.7)) .
$$

\section{GENERATING THE LENGTH DISTRIBUTION, THE LENGTH DIAGRAM, AND THE FIBROGRAM OF COTTON FIBRE}

The cotton length distribution by weight (obtained by measuring the weight of fibres in each length group) can be described by the following equation:

$$
f(l)= \begin{cases}\frac{w_{1}}{c} & \text { if } l \in[0, c] \\ \vdots & \\ \frac{w_{i}}{c} & \text { if } l \in[(i-1) c, i c] \\ \vdots & \\ \frac{w_{n}}{c} & \text { if } l \in[(n-1) c, n c] \\ 0 & \text { if } l \geq n c .\end{cases}
$$

$w_{1}, w_{2}, \ldots, w_{n}$ are the weight proportions of fibres, respectively, on the length groups $[0, c],[c, 2 c], \ldots,[(n-1) c, n c]$. Such $c$ is the length group width. For example, in the AFIS case $c$ is $2 \mathrm{~mm}$ and it is $2.5 \mathrm{~mm}$ for the Almeter.

The corresponding length diagram by weight $q$ can be obtained by summing $f$ from the longest to the shortest length by using (1):

$$
q(l)= \begin{cases}w_{1} \frac{c-l}{c}+\sum_{j=2}^{n} w_{j} & \text { if } l \in[0, c] \\ \vdots \\ w_{i} \frac{i c-l}{c}+\sum_{j=i+1}^{n} w_{j} & \text { if } l \in[(i-1) c, i c] \\ \vdots & \\ w_{n} \frac{n c-l}{c} & \text { if } l \in[(n-1) c, n c] \\ 0 & \text { if } l \geq n c .\end{cases}
$$

The fibrogram by weight is calculated by summing and normalising $q$ from the longest to the shortest length. Equation (2) can be used to calculate the fibrogram, so

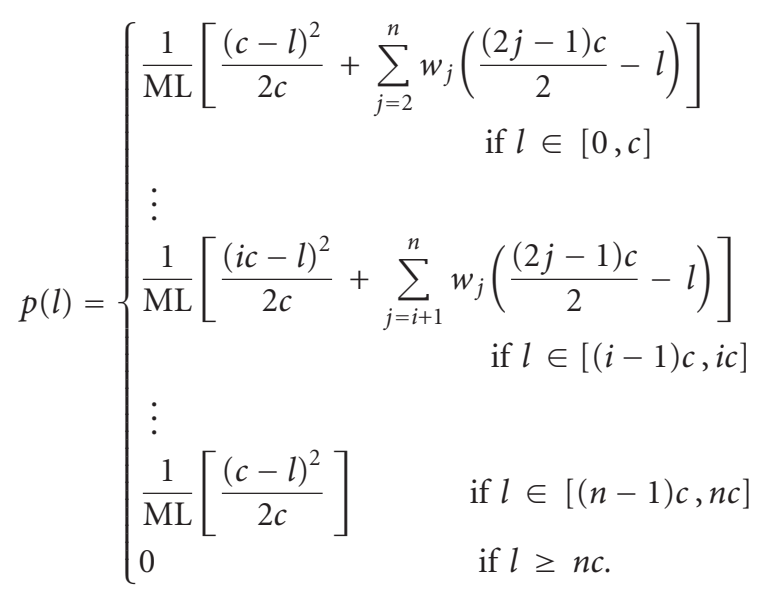




\section{GENERATING LENGTH DISTRIBUTION, LENGTH DIAGRAM, AND FIBROGRAM OF MULTICOMPONENT COTTON BLEND}

In this part of study, the length distribution, the length diagram, and the fibrogram of blend composed of $k$ different cottons with the proportions $x_{1}, x_{2}, \ldots, x_{k}$ will be generated.

Considering $k$ samples of different categories of fibres with respective masses $M_{1}, M_{2}, \ldots, M_{k}$ and with length distributions by weight $f_{1}, f_{2}, \ldots, f_{k}$, their length diagrams by weight are $q_{1}, q_{2}, \ldots, q_{k}$ and their fibrogram by weight are $p_{1}, p_{2}, \ldots, p_{k}$.

$m_{i}(l)$ and $f_{i}(l)$ are the weight and the proportion of the sample $i$ in the length group $[l-d l, l+d l]$, so

$$
f_{i}(l)=\frac{m_{i}(l)}{M_{i}} .
$$

The weight of the total blend is

$$
M=\sum_{i=1}^{k} M_{i} .
$$

The ratio of the sample $i$ in the blend is

$$
x_{i}=\frac{M_{i}}{M}
$$

The weight of the blend fibres that belong to the length group $[l-d l, l+d l]$ is $m(l)=\sum_{j=1}^{k} m_{i}$ and their percentage is $f(l)=$ $m(l) / M=\sum_{i=1}^{k} m_{i}(l) / M=\sum_{i=1}^{k}\left(m_{i}(l) / M_{i}\right)\left(M_{i} / M\right)$.

So according to (3) and (5),

$$
f(l)=\sum_{i=1}^{k} x_{i} f_{i}(l)
$$

The mean length of the blend is

$$
\mathrm{ML}=\int_{0}^{\infty} l f(l) d l=\int_{0}^{\infty}\left[\sum_{i=1}^{k} x_{i} f_{i}(l)\right] d l=\sum_{i=1}^{k}\left[x_{i} \int_{0}^{\infty} l f_{i}(l) d l\right],
$$

then

$$
\mathrm{ML}=\sum_{i=1}^{k} x_{i} \mathrm{ML}_{i}
$$

The length variance of the blend is (details of the derivations are given in the appendix)

$$
\sigma^{2}=\sum_{i=1}^{k} x_{i} \sigma_{i}^{2}+\sum_{1 \leq i<j \leq k} x_{i} x_{j}\left(\mathrm{ML}_{i}-\mathrm{ML}_{j}\right)^{2}
$$

The coefficient of the blend length variation is

$$
\begin{aligned}
\mathrm{CV} \%=\frac{1}{\sum_{i=1}^{k} x_{i} \mathrm{ML}_{i}}[ & \sum_{i=1}^{k} x_{i} \mathrm{ML}_{i}^{2} \mathrm{CV}_{i}^{2} \\
& \left.+\sum_{1 \leq i<j \leq k} x_{i} x_{j}\left(\mathrm{ML}_{i}-\mathrm{ML}_{j}\right)^{2}\right]^{1 / 2} .
\end{aligned}
$$

The biased-weight diagram of the blend is

$$
\begin{aligned}
q(l) & =\int_{l}^{\infty} f(t) d t=\int_{l}^{\infty}\left[\sum_{i=1}^{k} x_{i} f_{i}(t)\right] d t \\
& =\sum_{i=1}^{k}\left[x_{i} \int_{l}^{\infty} f_{i}(t) d t\right],
\end{aligned}
$$

then

$$
\begin{aligned}
q(l) & =\sum_{i=1}^{k} x_{i} q_{i}(l), \\
p(l) & =\frac{1}{\mathrm{ML}} \int_{l}^{\infty} q(t) d t=\frac{1}{\mathrm{ML}} \int_{l}^{\infty}\left[\sum_{i=1}^{k} x_{i} q_{i}(t)\right] d t \\
& =\frac{1}{\mathrm{ML}} \sum_{i=1}^{k}\left[x_{i} \int_{l}^{\infty} q_{i}(t) d t\right] \\
& =\sum_{i=1}^{k}\left[\frac{x_{i} \mathrm{ML}_{i}}{\mathrm{ML}} \frac{1}{\mathrm{ML}_{i}} \int_{l}^{\infty} q_{i}(t) d t\right],
\end{aligned}
$$

then

$$
p(l)=\sum_{i=1}^{k} \frac{x_{i} \mathrm{ML}_{i}}{\mathrm{ML}} p_{i}(l)
$$

The formulas (20), (26), and (27) showed the equations that relate ,respectively, the distribution $f$, the length diagram $q$, and the fibrogram $p$ of the blend to, respectively, the distributions $f_{1}, f_{2}, \ldots, f_{k}$, the length diagrams $q_{1}, q_{2}, \ldots, q_{k}$, and the fibrograms $p_{1}, p_{2}, \ldots, p_{k}$ of the $k$ components. These equations used with (14), (15), and (16) allow generating the equations of the length distribution, length diagram, and fibrogram of multicomponent cotton blend.

Thus

$$
f(l)= \begin{cases}\sum_{h=1}^{k} x_{h} \frac{w_{h 1}}{c} & \text { if } l \in[0, c] \\ \vdots & \\ \sum_{h=1}^{k} x_{h} \frac{w_{h i}}{c} & \text { if } l \in[(i-1) c, i c] \\ \vdots & \\ \sum_{h=1}^{k} x_{h} \frac{w_{h n}}{c} & \text { if } l \in[(n-1) c, n c] \\ 0 & \text { if } l \geq n c,\end{cases}
$$


TABLE 1: Length characteristics of cottons.

\begin{tabular}{|c|c|c|c|c|c|c|c|c|c|c|}
\hline Cotton category & $\mathrm{ML}(\mathrm{mm})$ & UQL $(\mathrm{mm})$ & UHML $(\mathrm{mm})$ & UQML $(\mathrm{mm})$ & $\mathrm{SL}_{50 \%}(\mathrm{~mm})$ & $\mathrm{SL}_{2.5 \%}(\mathrm{~mm})$ & $\mathrm{CV}(\%)$ & $\mathrm{UI}(\%)$ & $\mathrm{UR}(\%)$ & SFC(\%) \\
\hline $\mathrm{Eg}$ & 31.2 & 37.4 & 38.5 & 42.7 & 15.9 & 39.9 & 30.1 & 81 & 39.9 & 3.1 \\
\hline $\mathrm{Uz}$ & 26 & 30.6 & 32 & 35.5 & 13.2 & 33.8 & 30.5 & 81.4 & 39.1 & 4.5 \\
\hline USA1 & 30.2 & 35.4 & 36.7 & 40.5 & 15.3 & 38 & 28.1 & 82.2 & 40.3 & 2.5 \\
\hline USA2 & 24 & 28.9 & 29.9 & 32.9 & 12.4 & 31 & 31.6 & 80.5 & 39.9 & 7 \\
\hline $\mathrm{Br}$ & 25.6 & 31.3 & 32.5 & 36 & 13.2 & 34.1 & 33.9 & 79 & 38.6 & 7.1 \\
\hline
\end{tabular}

$W_{h i}$ is weight of fibres from cotton $h$ in the length class [ $i-$ 1) $c, i c]$,

$$
\begin{aligned}
& q(l)=\left\{\begin{array}{l}
\begin{array}{l}
\sum_{h=1}^{k} x_{h} w_{h 1} \frac{c-l}{c}+\sum_{j=2}^{n} \sum_{h=1}^{k} x_{h} w_{h j} \\
\quad \text { if } l \in[0, c]
\end{array} \\
\begin{array}{l}
\sum_{h=1}^{k} x_{h} w_{h i} \frac{i c-l}{c}+\sum_{j=i+1}^{n} \sum_{h=1}^{k} x_{h} w_{h j} \\
\vdots \\
\text { if } l \in[(i-1) c, i c]
\end{array} \\
\begin{array}{l}
\sum_{h=1}^{k} x_{h} w_{h n} \frac{n c-l}{c} \\
\text { if } l \in[(n-1) c, n c]
\end{array} \\
\text { if } l \geq n c,
\end{array}\right. \\
& \operatorname{MLp}(l)=\left\{\begin{array}{cc}
\frac{(c-l)^{2}}{2 c}+\sum_{j=2}^{n}[ & \left.\left(\frac{2 j-1}{2} c-l\right) \sum_{h=1}^{k} x_{h} w_{h j}\right] \\
& \text { if } l \in[0, c] \\
\frac{(i c-l)^{2}}{2 c}+\sum_{j=i+1}^{n}\left[\left(\frac{2 j-1}{2} c-l\right) \sum_{h=1}^{k} x_{h} w_{h j}\right] & \text { if } l \in[(i-1) c, i c] \\
\frac{(n c-l)^{2}}{2 c} & \text { if } l \in[(n-1) c, n c] \\
0 \quad & \text { if } l \geq n c .
\end{array}\right.
\end{aligned}
$$

\section{VARIATION OF STATISTICAL CHARACTERISTICS BY WEIGHT OF COTTON BINARY BLENDS}

\subsection{Materials and methods}

The length distribution by weight of five categories of cotton, where two (Egyptian (Eg) and USA1) are long and three
(Brazilian (Br), USA2, and Uzbekistani (Uz)) are medium length, was measured by AFIS. Their length parameters are given in Table 1. AFIS allows measuring the weight of fibres in each $2 \mathrm{~mm}$ length group.

Four binary blends were studied (USA2/Brazilian; Egyptian/USA1, USA1/USA2, and Egyptian/Uzbekistani).

Particularly for binary blends, the following equations (31), (32), and (33) of the length distribution, the length diagram, and the fibrogram by weight can be derived from (28), (29), and (30):

$$
f(l)= \begin{cases}x \frac{w_{11}}{c}+(1-x) \frac{w_{21}}{c} & \text { if } l \in[0, c] \\ \vdots & \\ x \frac{w_{1 i}}{c}+(1-x) \frac{w_{2 i}}{c} & \text { if } l \in[(i-1) c, i c] \\ \vdots & \text { if } l \in[(n-1) c, n c] \\ x \frac{w_{1 n}}{c}+(1-x) \frac{w_{2 n}}{c} & \text { if } l \geq n c,\end{cases}
$$

where $w_{1 i}$ is the weight of fibres from cotton 1 in the length group $[(i-1) c, i c]$ and $w_{2 i}$ is the weight of fibres from cotton 2 in the length group $[(i-1) c, i c]$ :

$$
q(l)=\left\{\begin{array}{rr}
\left(x w_{11}+(1-x) w_{21}\right) \frac{c-l}{c}+\sum_{j=2}^{n}\left(x w_{1 j}+(1-x) w_{2 j}\right) \\
\text { if } l \in[0, c] \\
\begin{array}{rl}
\left(x w_{1 i}+(1-x) w_{2 i}\right) \frac{i c-l}{c}+\sum_{j=i+1}^{n}\left(x w_{1 j}+(1-x) w_{2 j}\right) \\
\text { if } l \in[(i-1) c, i c] \\
\vdots & \text { if } l \in[(n-1) c, n c] \\
\left(x w_{1 n}+(1-x) w_{2 n}\right) \frac{n c-l}{c} & \text { if } l \geq n c,
\end{array}
\end{array}\right.
$$




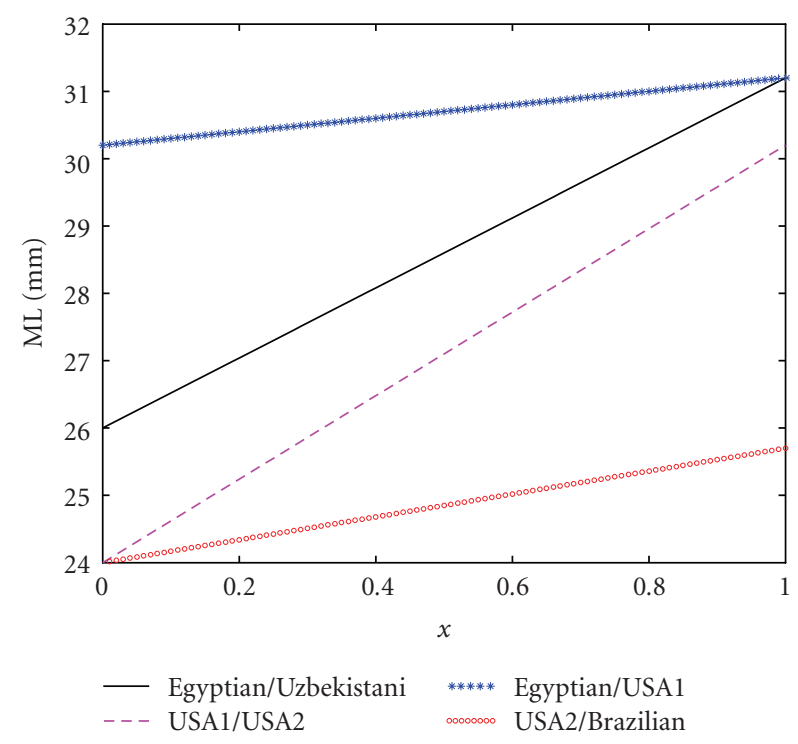

FIgURE 1: ML dependence to $x$.

$$
\operatorname{ML} p(l)= \begin{cases}\frac{(c-l)^{2}}{2 c}+\sum_{j=2}^{n}\left(\frac{2 j-1}{2} c-l\right)\left(x w_{1 j}+(1-x) w_{2 j}\right) \\ \text { if } l \in[0, c] \\ \frac{(i c-l)^{2}}{2 c}+\sum_{j=i+1}^{n}\left(\frac{2 j-1}{2} c-l\right)\left(x w_{1 j}+(1-x) w_{2 j}\right) \\ \text { if } l \in[(i-1) c, i c] \\ \frac{(n c-l)^{2}}{2 c} & \text { if } l \in[(n-1) c, n c] \\ 0 & \text { if } l \geq n c .\end{cases}
$$

The binary blend mean length ML and the coefficient of length variation $\mathrm{CV} \%$ are given by the following relationships. They are particular cases of (22) and (23);

$$
\begin{aligned}
\mathrm{ML}= & x \mathrm{ML}_{1}+(1-x) \mathrm{ML}_{2}, \\
\mathrm{CV} \%= & \frac{1}{x \mathrm{ML}_{1}+(1-x) \mathrm{ML}_{2}} \\
& \times\left[x \mathrm{ML}_{1}^{2} \mathrm{CV}_{1}^{2}+(1-x) \mathrm{ML}_{2}^{2} \mathrm{CV} \% \frac{2}{2}\right. \\
& \left.+x(1-x)\left(\mathrm{ML}_{1}-\mathrm{ML}_{2}\right)^{2}\right]^{1 / 2}
\end{aligned}
$$

where $\mathrm{ML}_{1}$ and $\mathrm{ML}_{2}$ are the mean lengths of the two cottons in the blend.

For each one of the binary blends, the ratios ( $x$ and (1$x)$ ) of the two components are varied from 0 to 1 by 0.01 step; the blend length distribution, length diagram, and fibrogram were calculated in each step by using (31), (32), and (33). The statistical parameters (ML, UQL, UHML, UQML, $\mathrm{SL}_{50 \%}, \mathrm{SL}_{2.5 \%}, \mathrm{CV} \%$, UI\%, UR\%, and SFC\%) were calculated

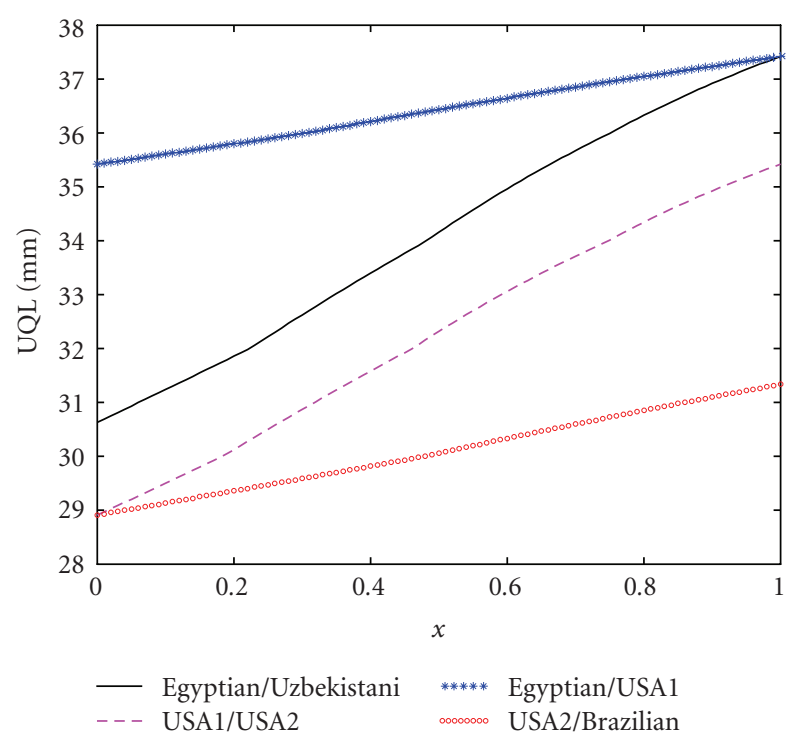

FIGURE 2: UQL dependence to $x$.

by using and resolving numerically (3), (6), (7), (8), (9), (10), (11), (12), and (13). The numerical resolution method consists to find the length corresponding to a minimum value of a difference. For example, to determine the parameter UQL (defined by $q(\mathrm{UQL})=0.25$ ), we vary the length $l$ from 0 to a maximum length $(60 \mathrm{~mm})$ and for each length value we calculate the difference $|q(\mathrm{UQL})-0.25|$. The length UQL corresponds to the minimum value of this difference.

Then the variation curve of each length parameters, for example, UQL dependence on $x$ can be plotted. After that, this curve is compared to linear variation by calculating the residual DUQL $=\mathrm{UQL}-\left(x \mathrm{UQL}_{1}+(1-x) \mathrm{UQL}_{2}\right)$, where $\mathrm{UQL}_{1}$ and $\mathrm{UQL}_{2}$ are the corresponding parameters of the two components, the variations of the residuals dependence on $x$ are plotted too.

\section{RESULTS AND DISCUSSIONS}

For the four binary blends, the variations of each length parameters and the residuals to the linear variation versus the ratio $x$ of the first component are plotted and represented in Figures 1-18.

\subsection{Mean length by weight (ML)}

The mean length (ML) of the blend was calculated by using (3). As shown in Figure 1, its variation dependence to $x$ is linear. This confirms the results proved in (22).

\subsection{Upper quartile length by weight (UQL)}

The upper quartile length UQL was calculated by resolving numerically the equation $q(l)=0.25$ (we increment $l$ by $0.01 \mathrm{~mm}$ and we keep the length value corresponding to a minimum value of $|q(l)-0.25|)$. 


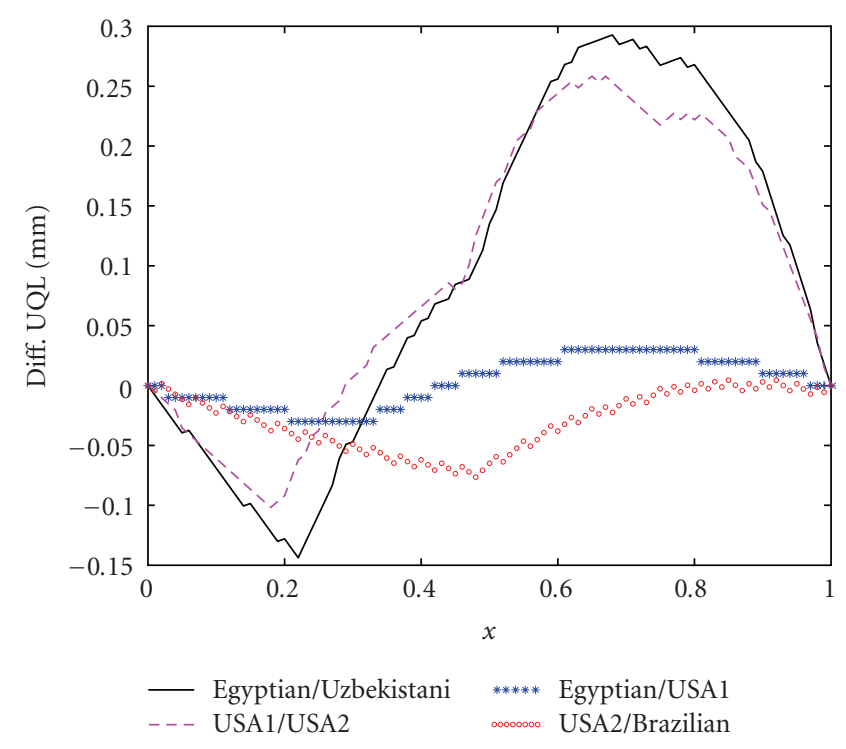

Figure 3: DUQL dependence to $x$.

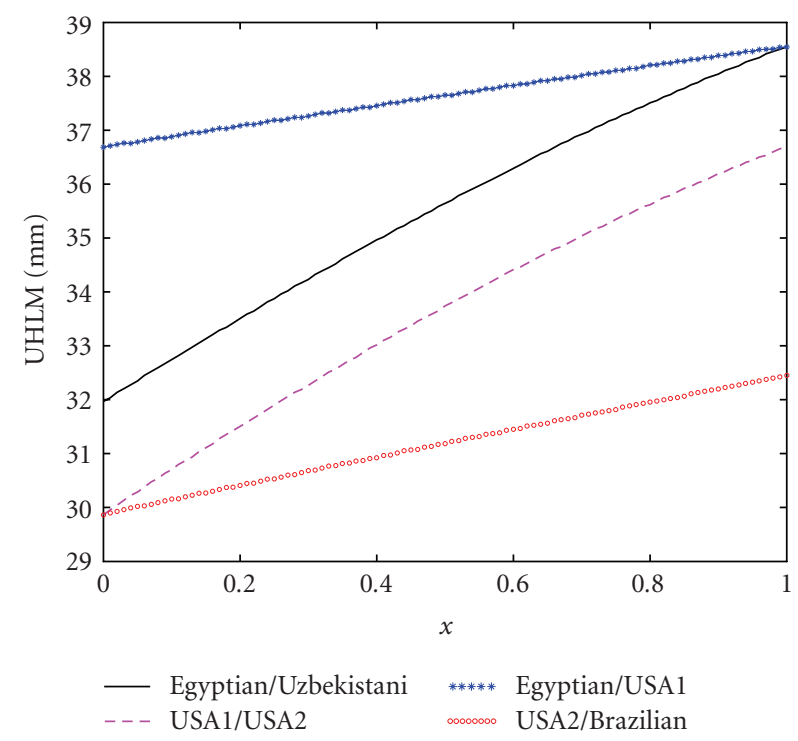

Figure 4: UHML dependence to $x$.

Figures 2 and 3 show that for the blends USA2 (medium)/Brazilian (medium) and Egyptian (long)/USA1 (long) the variation of UQL is nearly linear. For the blends USA1 (long)/USA2 (medium) and Egyptian (long)/Uzbekistani (medium), the variation is little sigmoid but the absolute value of DUQL is always lower than $0.3 \mathrm{~mm}$.

\subsection{Upper half mean length by weight (UHML)}

The upper half mean length is calculated by using (8). For the blends USA2 (medium)/Brazilian (medium) and Egyptian (long)/USA1 (long), the variation of UHML is nearly linear expressed by a DUHML variation close to zero. For the blends USA1 (long)/USA2 (medium) and Egyptian

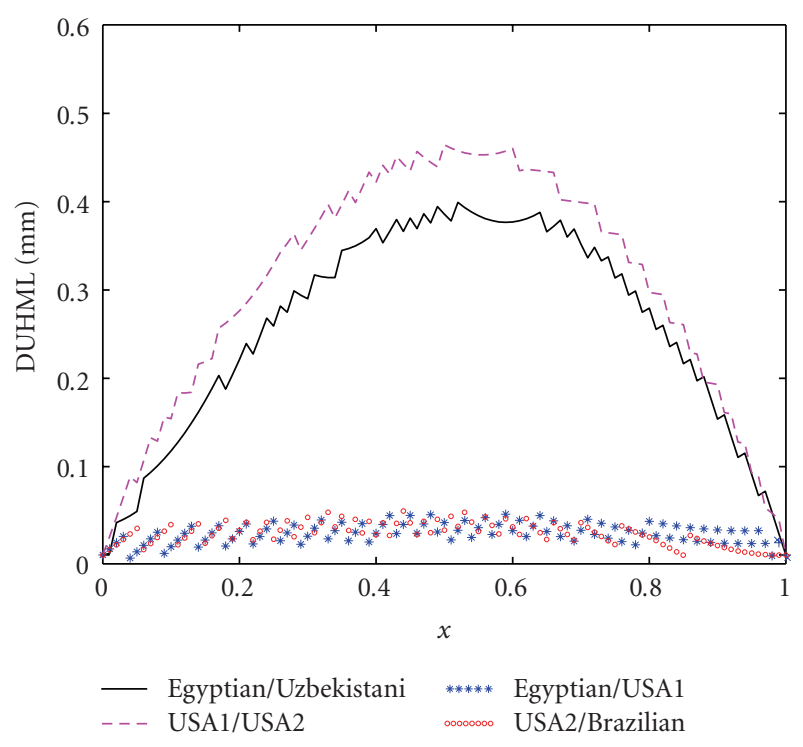

Figure 5: DUHML dependence to $x$.

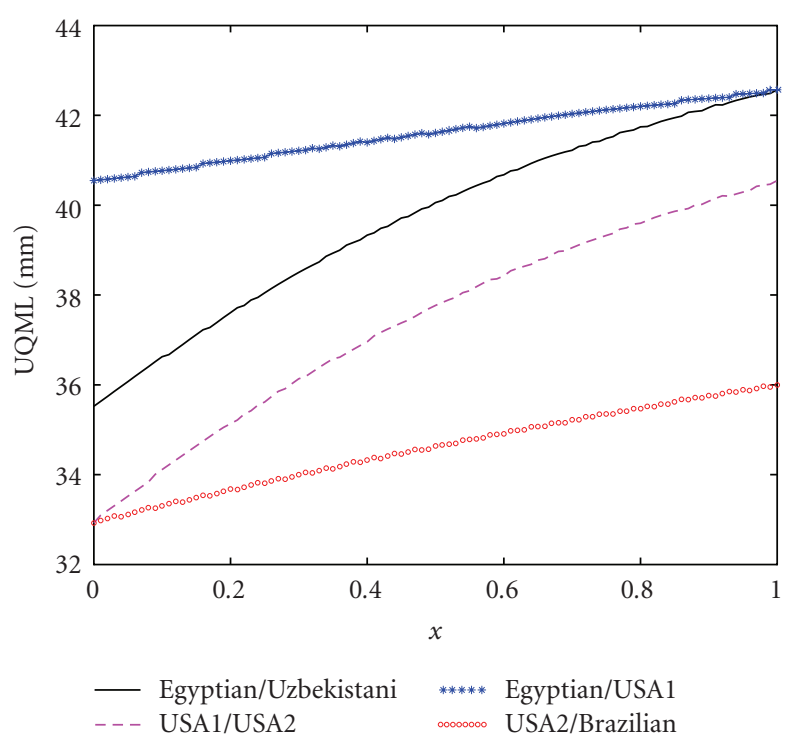

FIgURE 6: UQML dependence to $x$.

(long)/Uzbekistani (medium), the variation is less linear and DUHML can be equal to $0.5 \mathrm{~mm}$ when the two components have compared ratios in the blend.

\subsection{Upper quarter mean length by weight (UQML)}

The upper quarter mean length is calculated by using (9). For the blends USA2 (medium)/Brazilian (medium) and Egyptian (long)/USA1 (long), the variation of UQML is nearly linear and DUQML is less than $0.2 \mathrm{~mm}$. For the blends USA1 (long)/USA2 (medium) and Egyptian (long)/Uzbekistani (medium), the variation is nonlinear and DUQML can extend $1 \mathrm{~mm}$ when the two components have compared ratios in the blend. 


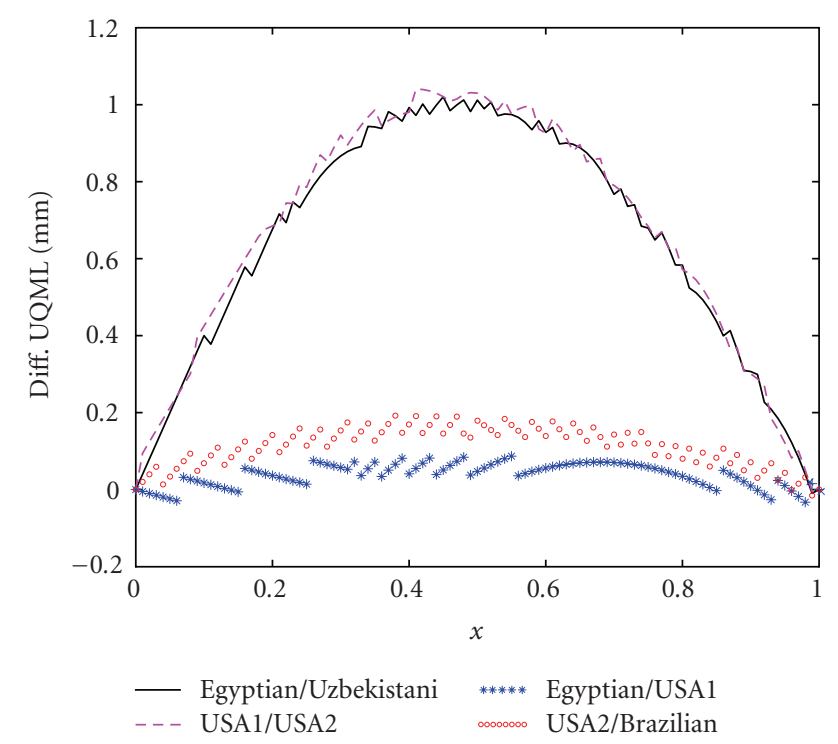

FIGURE 7: DUQML dependence to $x$.

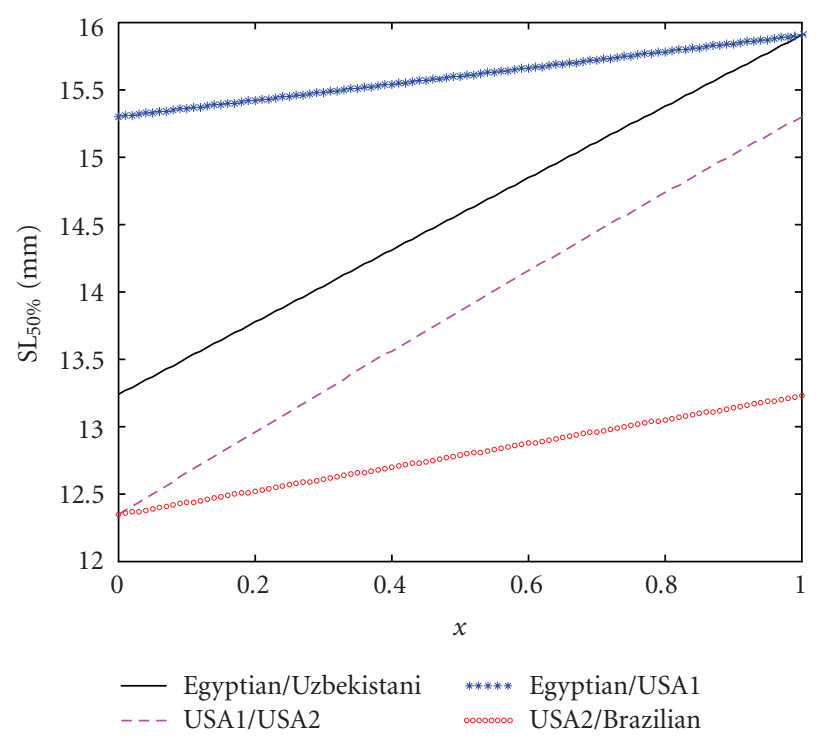

Figure 8: $\mathrm{SL}_{50 \%}$ dependence to $x$.

\subsection{Span length by weight $\left(S L_{50 \%}\right)$}

The $50 \%$ span length is calculated by resolving numerically the equation $p(l)=0.5$. Its variation dependence on $x$ can be considered as linear for the four blends expressed by DSL $_{50 \%}$ inferior to $0.05 \mathrm{~mm}$.

\subsection{Span length by weight $\left(S L_{2.5 \%}\right)$}

The $2.5 \%$ span length is calculated by a numeric resolution of the equation $p(l)=0.025$. The variation of this parameter is nearly linear for the USA2 (medium)/Brazilian (medium) and Egyptian (long)/USA1 (long) blends. But

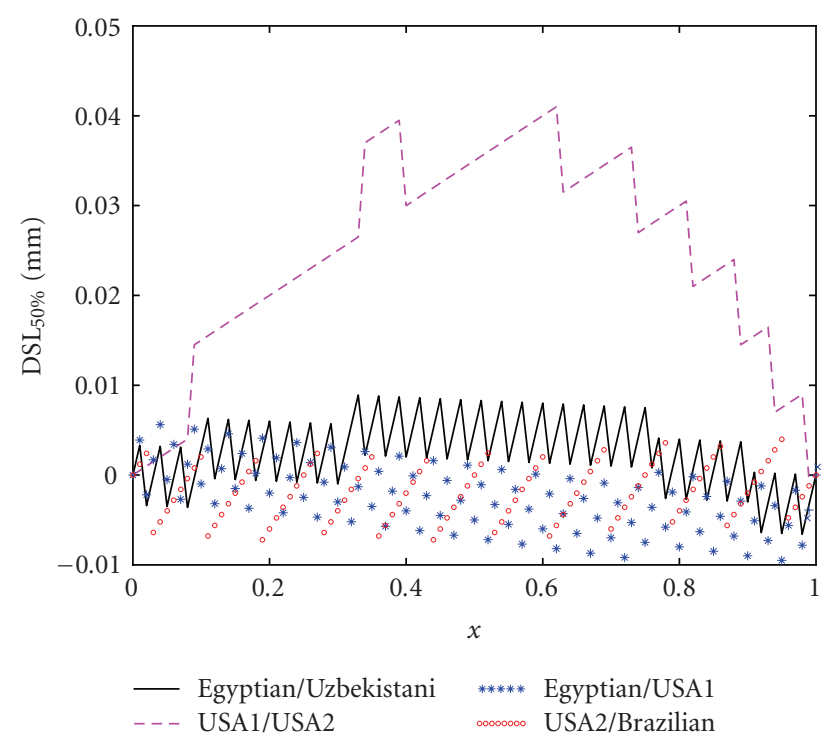

Figure 9: DSL $_{50 \%}$ dependence to $x$.

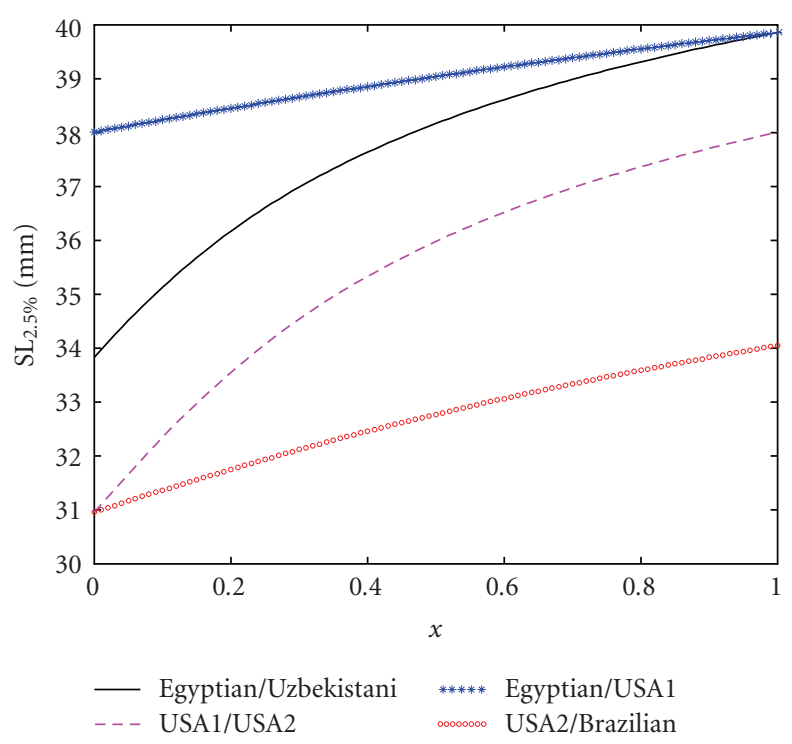

FIgURE 10: $\mathrm{SL}_{2.5 \%}$ dependence to $x$.

for the blends USA1 (long)/USA2 (medium) and Egyptian (long)/Uzbekistani (medium), the variation is nonlinear; $\mathrm{DSL}_{2.5 \%}$ is always positive and it can extend $1.5 \mathrm{~mm}$.

\subsection{Coefficient of fibre length variation by weight (CV\%)}

The coefficient of length variation $\mathrm{CV} \%$ was calculated by using (6), where $\sigma$ was calculated by using (4) and (5).

Figure 12 shows that the CV\% of the blends USA1 (long)/USA2 (medium) and Egyptian (long)/Uzbekistani (medium) has a convex variation curve and it can be higher than the two components CV\%. But for the blends USA2 


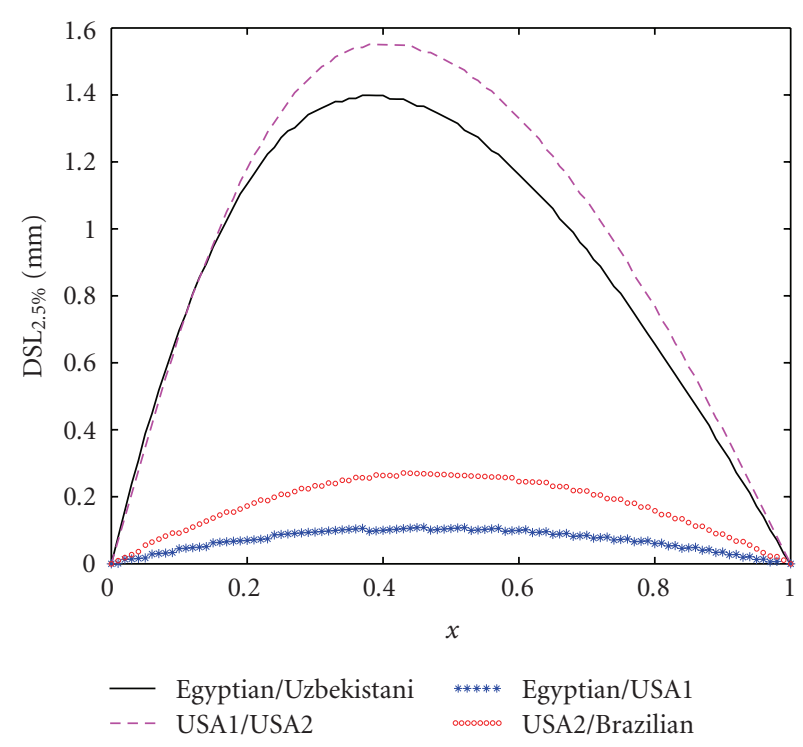

Figure 11: DSL $2.5 \%$ dependence to $x$.

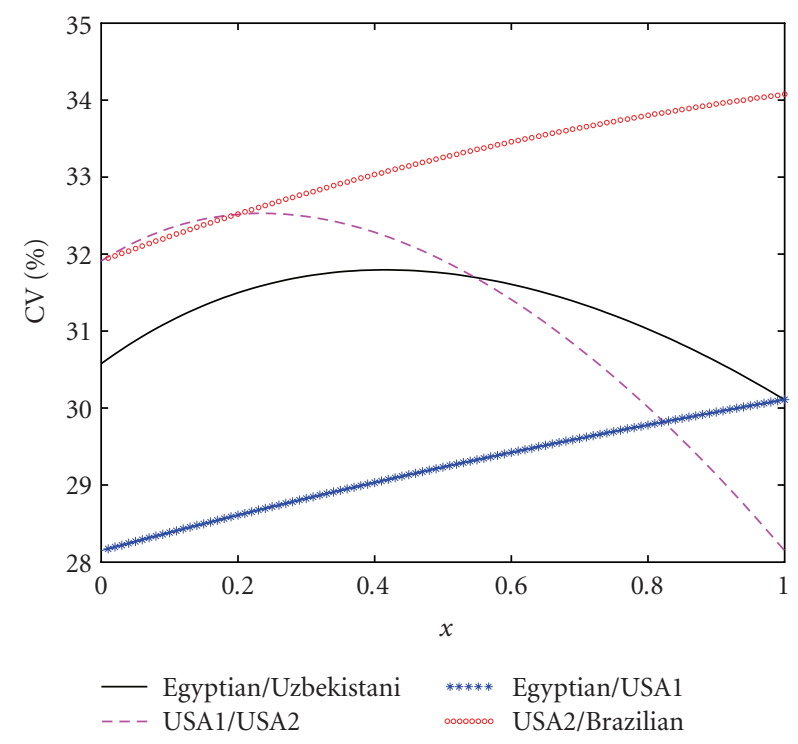

FIgURE 12: CV\% dependence to $x$.

(medium)/Brazilian (medium) and Egyptian (long)/USA1 (long), its variation is close to a right line. Equation (35) proves this result. When the two cottons have close mean lengths, the quadratic term $\left(\mathrm{ML}_{1}-\mathrm{ML}_{2}\right)^{2} x(1-x)$ is negligible.

\subsection{Uniformity index (UI\%)}

The uniformity index is calculated by using (11). DUI\% is negative and it is nearly zero for the USA2 (medium)/Brazilian (medium) and Egyptian (long)/USA1 (long) blends. So the variation of this parameter can be considered linear in the case of these two blends, and it can reach $-1 \%$ (more

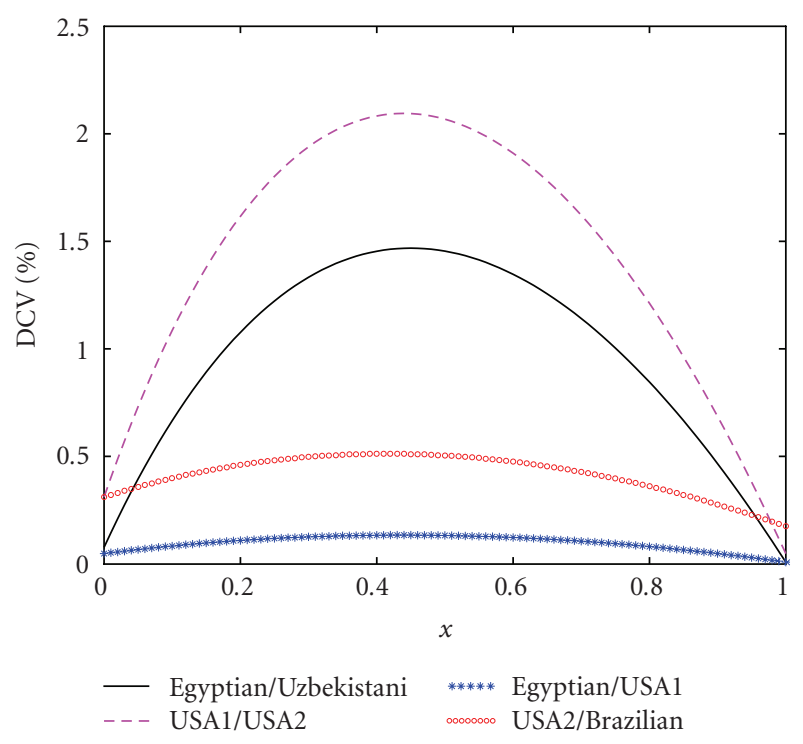

Figure 13: DCV\% dependence to $x$.

than $50 \%$ of the difference between the UI\% of the two components) in the two blends, USA1 (long)/USA2 (medium) and Egyptian (long)/Uzbekistani (medium).

\subsection{Uniformity ratio (UR\%)}

The uniformity ratio is calculated by using (12). As DUI\% and DUR\% are negative, they are close to zero for the USA2 (medium)/Brazilian (medium) and Egyptian (long)/USA1 (long) blends. For the USA1 (long)/USA2 (medium) and Egyptian (long)/ Uzbekistani (medium) blends, the variation of UR $\%$ is nonlinear and the absolute value of DUR\% can extend $1.5 \%$. This is more than $100 \%$ of the difference between the UR\% of the two components.

\subsection{Short fibre content (SFC\%)}

The short fibre content was calculated by using (13). As shown in Figure 18, the variation of this parameter is linear. That can be mathematically proved:

$$
\begin{aligned}
\mathrm{SFC} & =\int_{0}^{12.7} f(l) d l=\int_{0}^{12.7}\left[x f_{1}(l)+(1-x) f_{2}(l)\right] d l \\
& =x \int_{0}^{12.7} f_{1}(l) d l+(1-x) \int_{0}^{12.7} f_{2}(l) d l \\
& =x \mathrm{SFC}_{1}+(1-x) \mathrm{SFC}_{2} .
\end{aligned}
$$

\section{COMPARISON TO REAL BLENDS}

We tried to compare the variation of the statistical length parameters determined from blend distribution functions expressed previously from the ones of real blends.

The USA1/USA2 binary blend studied above was considered. Nine binary blends $(0.1 / 0.9,0.2 / 0.8,0.3 / 0.7,0.4 / 0.6$, $0.5 / 0.5,0.6 / 0.4,0.7 / 0.3,0.8 / 0.2$, and $0.9 / 0.1)$ were achieved and homogenised with manual method. The two ratios of 


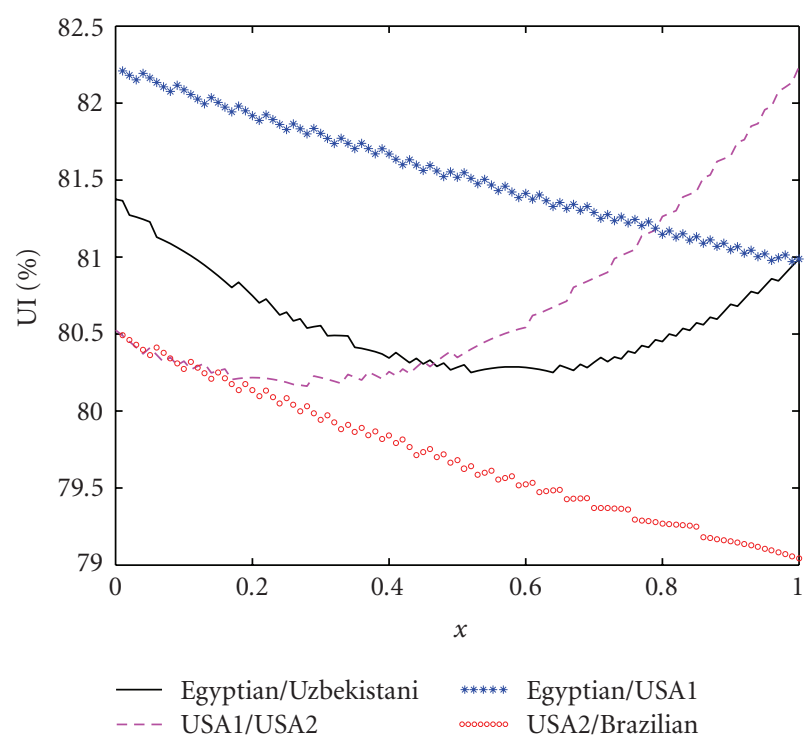

FIGURE 14: UI\% dependence to $x$.

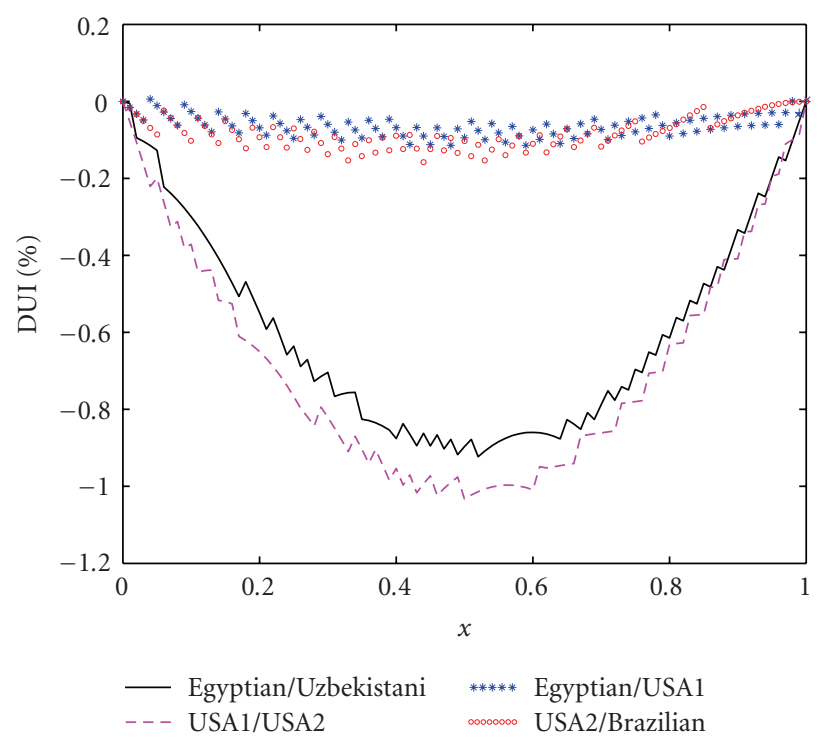

FIgURE 15: DUI\% dependence to $x$.

cottons composing the blend were obtained according to the French Norm (NFG 07062). Next, the cotton proportions are mixed using a manual method based on blend principle in spinning process. In order to have a homogenous blend in the transverse and longitudinal directions, a random meeting of fractions was done. The adopted method to achieve a binary blend (with $20 \mathrm{~g}$ weight) was as follows:

(1) sampling a weight $m_{i}$ of each constituent cotton respecting the proportions in the blend,

(2) dividing the weight $m_{i}$ in 16 equal fractions,

(3) using a random numbers table to gather 2 by 2 the fractions of the first cotton with those of the second,

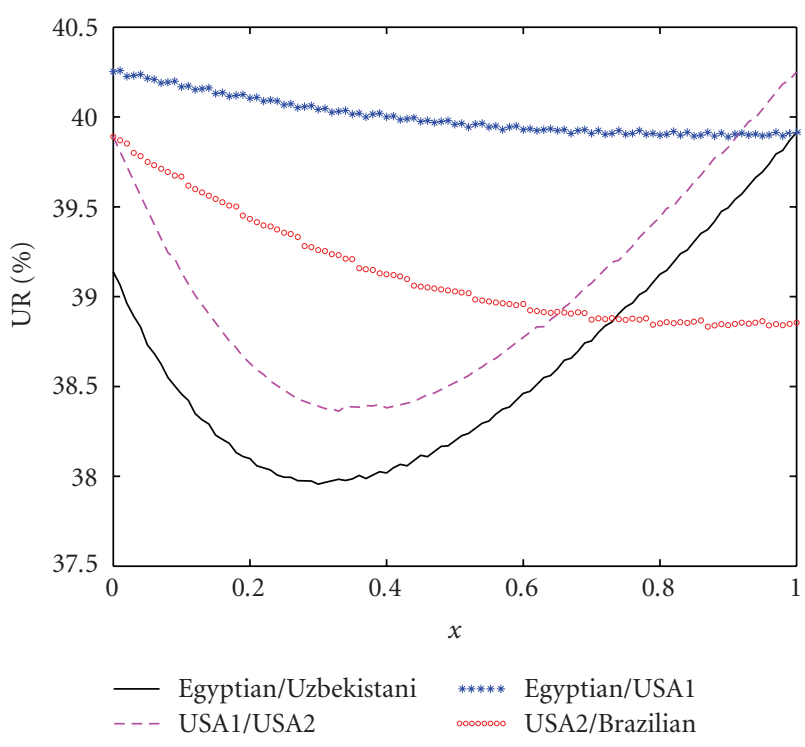

Figure 16: UR\% dependence to $x$.

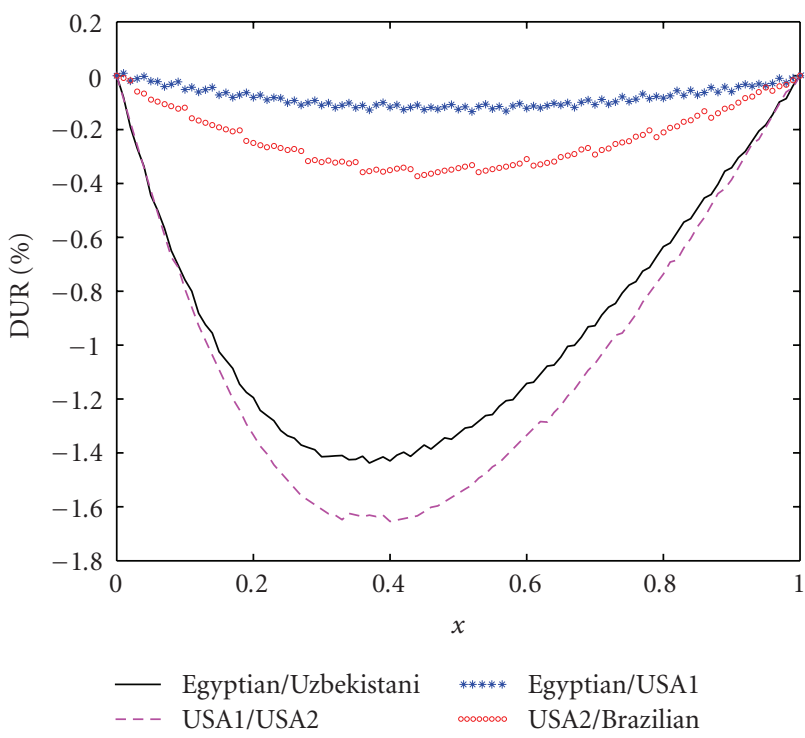

Figure 17: DUR\% dependence to $x$.

(4) the 16 resulting couples were divided in small tufts weighting less than $0.5 \mathrm{~g}$. Next, they were randomly mixed, then transformed manually into 6 slivers that will successively be doubled and stretched,

(5) every blended couple was divided again in two portions then subjected to steps 3 and 4 for three times.

Then, the length distributions of these nine blends were measured by AFIS. From these distributions, the length diagrams, the fibrograms, and the length characteristics were determined.

The correlation between the length parameter values of real blends and those determined from the length function distributions was calculated. 


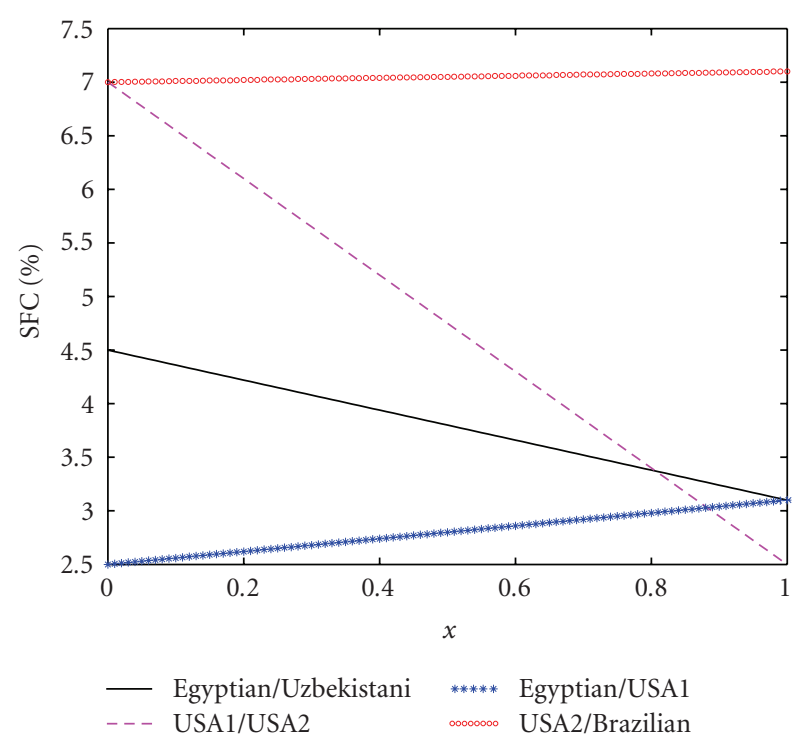

Figure 18: SFC\% dependence to $x$.

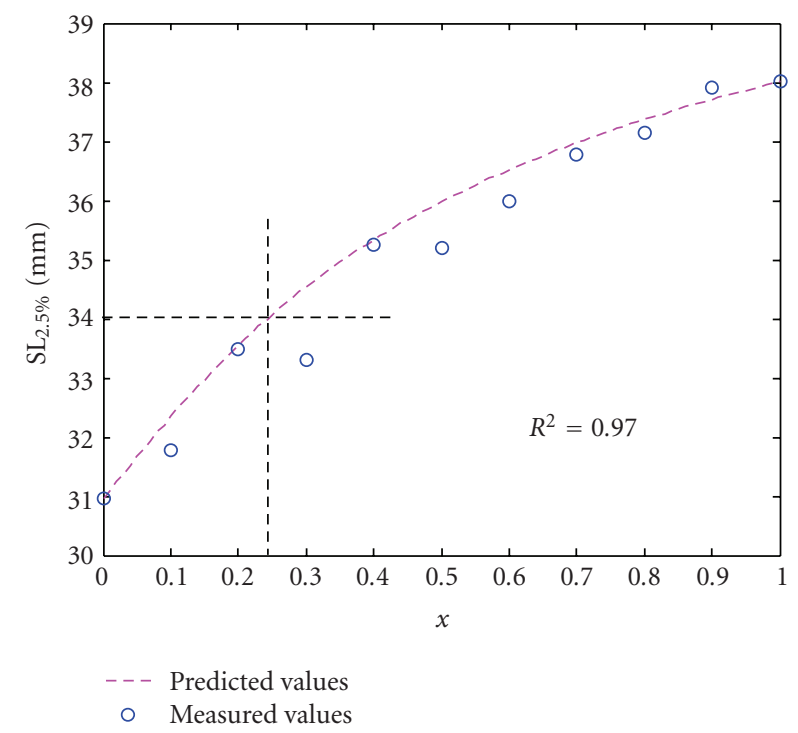

FIGURE 19: $\mathrm{SL}_{2.5 \%}$ values determined from established distribution functions and those of real blends.

For the two length parameters $\mathrm{SL}_{2.5 \%}$ and UR\%, the correlations are shown, respectively, in Figures 19 and 20.

Table 2 gives the determination coefficients for the other parameters.

Good correlations were obtained between the measured blend characteristics and those obtained from the established distribution functions. The determination coefficient $R^{2}$ varies between 0.79 and 0.97 for the different parameters. This result confirms, also, that the variation of the length characteristics according to the proportions of the components in the blend is not usually linear.

Nevertheless, the selection of cotton blends could not be done by using the linear programming method as usually done by the spinners and as in ELmoghazy study [12]. We

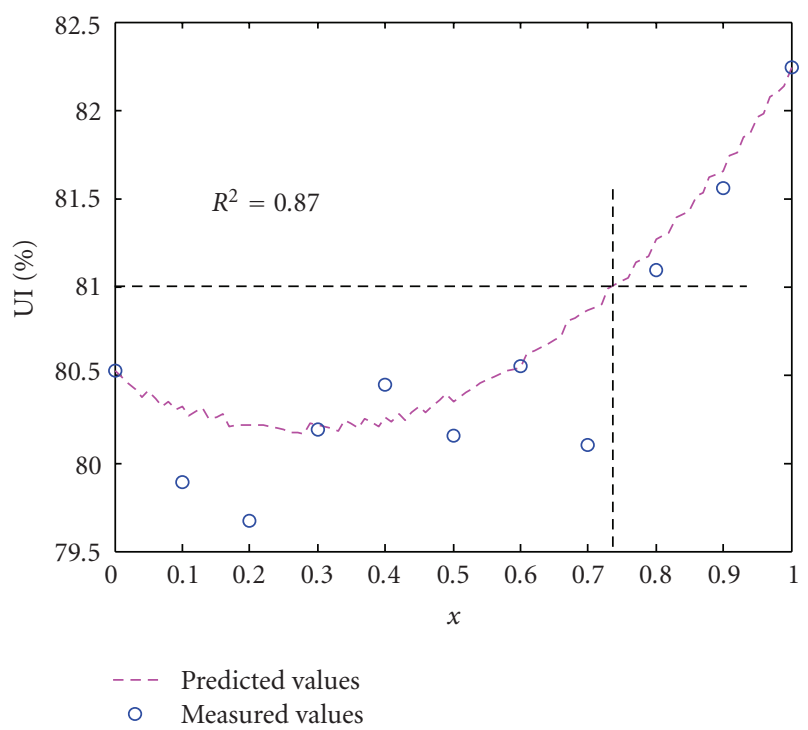

FIGURE 20: UI\% values determined from established distribution functions and those of real blends.

will prove that the selection of optimal blends based on the linear variation of the parameters and the selection based on the variation of the parameters showed in this study give different results.

As an example, we will try to select an optimal binary blend composed of the USA1 and USA2 cottons given in Table 1.

An optimal blend would verify the two following conditions:

$$
\begin{aligned}
\mathrm{SL}_{2.5 \%} & \geq 34 \mathrm{~mm}, \\
\mathrm{UI} \% & \geq 81 \% .
\end{aligned}
$$

The selection of an optimal USA1/USA2 blend by using the linear programming method is based on the resolution of the following system:

$$
\begin{array}{r}
38 x+31(1-x) \geq 34 \\
82.2 x+80.5(1-x) \geq 81
\end{array}
$$

The resolution of the system (38) gives what follows as solutions.

For all blends with the percentage of the USA1 cotton $x$ superior to 0.43 , the first condition $\left(\mathrm{SL}_{2.5 \%} \geq 34 \mathrm{~mm}\right)$ is verified.

For all blends with the percentage of the USA1 cotton $x$ superior to 0.29 , the second condition (UI\% $\geq 81 \%$ ) is verified.

However, only the blends with $x$ superior to 0.43 verify the two conditions.

So supposing that the variation of length parameters is linear, all blends with $x$ superior to 0.43 are optimal.

Our purpose is to use the variation of the two parameters $\mathrm{SL}_{2.5 \%}$ and UI\% curves shown in Figures 19 and 20. The graphic resolution of the system (36) gives what follows as solutions. 
TABle 2: Determination coefficients between the parameters determined from the blends established models and the real ones.

\begin{tabular}{|c|c|c|c|c|c|c|c|c|}
\hline Parameters & $\mathrm{ML}(\mathrm{mm})$ & UQL (mm) & UHML (mm) & UQML (mm) & $\mathrm{SL}_{50 \%}(\mathrm{~mm})$ & $\mathrm{CV}(\%)$ & $\mathrm{UR}(\%)$ & $\mathrm{SFC}(\%)$ \\
\hline Determination coefficient $\left(R^{2}\right)$ & 0.83 & 0.89 & 0.93 & 0.94 & 0.84 & 0.86 & 0.79 & 0.86 \\
\hline
\end{tabular}

For all blends with the percentage of the USA1 cotton $x$ superior to 0.24 , the first condition $\left(\mathrm{SL}_{2.5 \%} \geq 34 \mathrm{~mm}\right)$ is verified.

For all blends with the percentage of the USA1 cotton $x$ superior to 0.74 , the second condition (UI\% $\geq 81 \%$ ) is verified.

However, only the blends with $x$ superior to 0.74 verify the two conditions.

Based on the good correlation between our parameter variations and the measured blend parameters, the established length parameters given in this study allow selecting optimal length blends with a better precision than linear method usually used by the spinners.

\section{CONCLUSIONS}

First, we presented equations generating the cotton length distribution, the length diagram, and the fibrogram from a measured length distribution by weight.

Then the study was intended to put the concept of fibre length distribution, length diagram, and fibrogram by weight of a multicomponent blend of cottons in a perspective to show the variation of many statistical length parameters according to the cotton categories and their ratios in the blend, with a particular emphasis on the variation of binary blend parameters.

A multicomponents blend of $n$ cotton categories can be considered as a binary blend of one cotton with a blend of $n-1$ cottons. So such a study can be valuable for blends of more than two cottons.

The variations of the mean length (ML 50\%), span length $\left(\mathrm{SL}_{50 \%}\right)$, and the short fibre content (SFC\%) dependence on the mass fraction of cottons in the blend are linear.

The variations of the other parameters such as the upper quartile length (UQL), the upper half mean length (UHML), the upper quarter mean length (UQML), the $2.5 \%$ span length $\mathrm{SL}_{2.5 \%}$, the coefficient of length variation $(\mathrm{CV} \%)$, the uniformity index (UI\%), and the uniformity ratio (UR\%) are not linear and the curves are more bent in the case of blends constituted from long cottons and medium length ones. But the variation of these parameters is considered to be linear when the two cottons in the blend have closed lengths.

We think that representation of the curves of the length parameter variations may allow the searcher to adjust these variation curves by analytic models that generate each length parameter. We already begin this work and we try to adjust cotton length distribution by theoretical blend distributions, from these distributions we are developing equations that relate all length parameters to only the statistic characteristics of these distributions.
The blend length parameters determined from the established blend distribution functions are compared to real blend parameters and good correlations are obtained.

Then the generation of the variation of length parameters allows predicting the blend characteristics and selecting the optimal blends with better precision than linear models. This result is now used, in a work in progress, with mathematical and statistical models established to estimate the other cotton blend parameters (fineness, maturity, strength, and elongation), to optimise the selection of multicomponent cotton blends by using multiobjectives optimisation techniques.

\section{APPENDIX}

We have

$$
\begin{aligned}
\sigma^{2} & =\int_{0}^{\infty}[t-\mathrm{ML}]^{2} f(t) d t \\
& =\int_{0}^{\infty} t^{2} f(t) d t-2 \mathrm{ML} \int_{0}^{\infty} t f(t) d t+\mathrm{ML}^{2} \int_{0}^{\infty} f(t) d t \\
& =\int_{0}^{\infty} t^{2} f(t) d t-\mathrm{ML}^{2}
\end{aligned}
$$

because $\int_{0}^{\infty} f(t) d t=1$ and $\int_{0}^{\infty} t f(t) d t=\mathrm{ML}$,

$$
\begin{aligned}
\sigma^{2} & =\int_{0}^{\infty} t^{2}\left[\sum_{i=1}^{k} x_{i} f_{i}(t)\right] d t-\left[\sum_{i=1}^{k} x_{i} \mathrm{ML}_{i}\right]^{2} \\
& =\sum_{i=1}^{k} x_{i} \int_{0}^{\infty} t^{2} f_{i}(t)-\left[\sum_{i=1}^{k} x_{i}^{2} \mathrm{ML}_{i}^{2}+2 \sum_{1 \leq i<j \leq k} x_{i} x_{j} \mathrm{ML}_{i} \mathrm{ML}_{j}\right], \\
\sigma^{2} & =\sum_{i=1}^{k} x_{i} \int_{0}^{\infty} t^{2} f_{i}(t)-\sum_{i=1}^{k} x_{i}^{2} \mathrm{ML}_{i}^{2}-2 \sum_{1 \leq i<j \leq k} x_{i} x_{j} \mathrm{ML}_{i} \mathrm{ML}_{j} .
\end{aligned}
$$

Knowing that $\sum_{i=1}^{k} x_{i}=1$ develops the term

$$
\begin{aligned}
\sum_{i=1}^{k} x_{i}^{2} \mathrm{ML}_{i}^{2} & =\sum_{i=1}^{k} x_{i}\left(1-\sum_{1 \leq j \neq i \leq k} x_{j}\right) \mathrm{ML}_{i}^{2} \\
& =\sum_{i=1}^{k} x_{i} \mathrm{ML}_{i}^{2}-\sum_{1 \leq j<i \leq k} x_{i} x_{j} \mathrm{ML}_{i}^{2}-\sum_{1 \leq j>i \leq k} x_{i} x_{j} \mathrm{ML}_{i}^{2} .
\end{aligned}
$$

We can write

$$
\sum_{1 \leq j<i \leq k} x_{i} x_{j} \mathrm{ML}_{i}^{2}=\sum_{1 \leq i<j \leq k} x_{i} x_{j} \mathrm{ML}_{j}^{2}
$$


Thus

$$
\begin{aligned}
\sum_{i=1}^{k} x_{i}^{2} \mathrm{ML}_{i}^{2} & =\sum_{i=1}^{k} x_{i}\left(1-\sum_{1 \leq j \neq i \leq k} x_{j}\right) \mathrm{ML}_{i}^{2} \\
& =\sum_{i=1}^{k} x_{i} \mathrm{ML}_{i}^{2}-\sum_{1 \leq i<j \leq k} x_{i} x_{j}\left(\mathrm{ML}_{i}^{2}+\mathrm{ML}_{j}^{2}\right),
\end{aligned}
$$

then

$$
\begin{aligned}
\sigma^{2}= & \sum_{i=1}^{k} x_{i} \int_{0}^{\infty} t^{2} f_{i}(t)-\sum_{i=1}^{k} x_{i} \mathrm{ML}_{i}^{2}-\sum_{1 \leq i<j \leq k} x_{i} x_{j}\left(\mathrm{ML}_{i}^{2}+\mathrm{ML}_{j}^{2}\right) \\
& -2 \sum_{1 \leq i<j \leq k} x_{i} x_{j} \mathrm{ML}_{i} \mathrm{ML}_{j}, \\
\sigma^{2}= & \sum_{i=1}^{k} x_{i}\left[\int_{0}^{\infty} t^{2} f_{i}(t) d t-\mathrm{ML}_{i}^{2}\right] \\
& -\sum_{1 \leq i<j \leq k} x_{i} x_{j}\left(\mathrm{ML}_{i}^{2}+\mathrm{ML}_{j}^{2}-2 \mathrm{ML}_{i} \mathrm{ML}_{j}\right) .
\end{aligned}
$$

Finally,

$$
\sigma^{2}=\sum_{i=1}^{k} x_{i} \sigma_{i}^{2}+\sum_{1 \leq i<j \leq k} x_{i} x_{j}\left(\mathrm{ML}_{i}-\mathrm{ML}_{j}\right)^{2} .
$$

[10] Y. E. Elmoghazy and Y. Gowayed, "Theory and practice of cotton fiber selection - part I: fiber selection techniques and bale picking algorithms," Textile Research Journal, vol. 65, no. 1, pp. 32-40, 1995.

[11] Y. E. El Mogahzy and Y. Gowayed, "Theory and practice of cotton fiber selection-part II: sources of cotton mix variability and critical factors affecting it," Textile Research Journal, vol. 65, no. 2, pp. 75-84, 1995.

[12] Y. E. El Mogahzy, "Optimizing cotton blend costs with respect to quality using HVI fiber properties and linear programming-part 1: fundamentals and advanced techniques of linear programming," Textile Research Journal, vol. 62, no. 1, pp. 1-8, 1992.

\section{AUTHOR CONTACT INFORMATION}

B. Azzouz: Textile Research Unit of ISET K-H, BP 68, Ksar Hellal 5070, Tunisia; azzouzbechiir@yahoo.fr

M. Ben Hassen: Textile Research Unit of ISET K-H, BP 68, Ksar Hellal 5070, Tunisia; benrayen@yahoo.fr

F. Sakli: Textile Research Unit of ISET K-H, BP 68, Ksar Hellal 5070, Tunisia; faouzi.sakli@isetkh.rnu.tn

faouzi.sakli@isetkh.rnu.tn

\section{REFERENCES}

[1] K. L. Hertel, "A method of fiber-length analysis using the fibrograph," Textile Research Journal, vol. 10, no. 12, pp. 510-525, 1940.

[2] C. B. Landstreet, "The fibrogram: its concept and use in measuring cotton fiber length," Textile Bulletin, vol. 87, no. 4, pp. 54-57, 1961.

[3] R. S. Krowicki, J. M. Hemstreet, and K. E. Duckett, "Different approach to generating the fibrogram from fiber-lengtharray data-part I: theory," Journal of the Textile Institute. Part 1, vol. 88, no. 1, part 1, pp. 1-4, 1997.

[4] R. S. Krowicki, J. M. Hemstreet, and K. E. Duckett, "A different approach to generating the fibrogram from fiber-length-array data-part II: application," Journal of the Textile Institute. Part 1, vol. 89, no. 1, pp. 1-9, 1998.

[5] R. S. Krowicki and K. E. Duckett, "An examination of the fibrogram," Textile Research Journal, vol. 57, no. 4, pp. 200-204, 1987.

[6] M. I. Zeidman, S. K. Batra, and P. E. Sasser, "Determining short fiber content in cotton-part I: some theoretical fundamentals," Textile Research Journal, vol. 61, no. 1, pp. 21-30, 1991.

[7] M. I. Zeidman, S. K. Batra, and P. E. Sasser, "Determining short fiber content in cotton-part II: measures of SFC from HVI data—statistical models," Textile Research Journal, vol. 61, no. 2, pp. 106-113, 1991.

[8] M. Zeidman and P. S. Sawhney, "Influence of fiber length distribution on strength efficiency of fibers in yarn," Textile Research Journal, vol. 72, no. 3, pp. 216-220, 2002.

[9] X. Cui, T. A. Calamari Jr., and M. W. Suh, "Theoritical and practical aspects of fiber length comparisons of various cottons," Textile Research Journal, vol. 68, no. 7, pp. 467-472, 1998. 

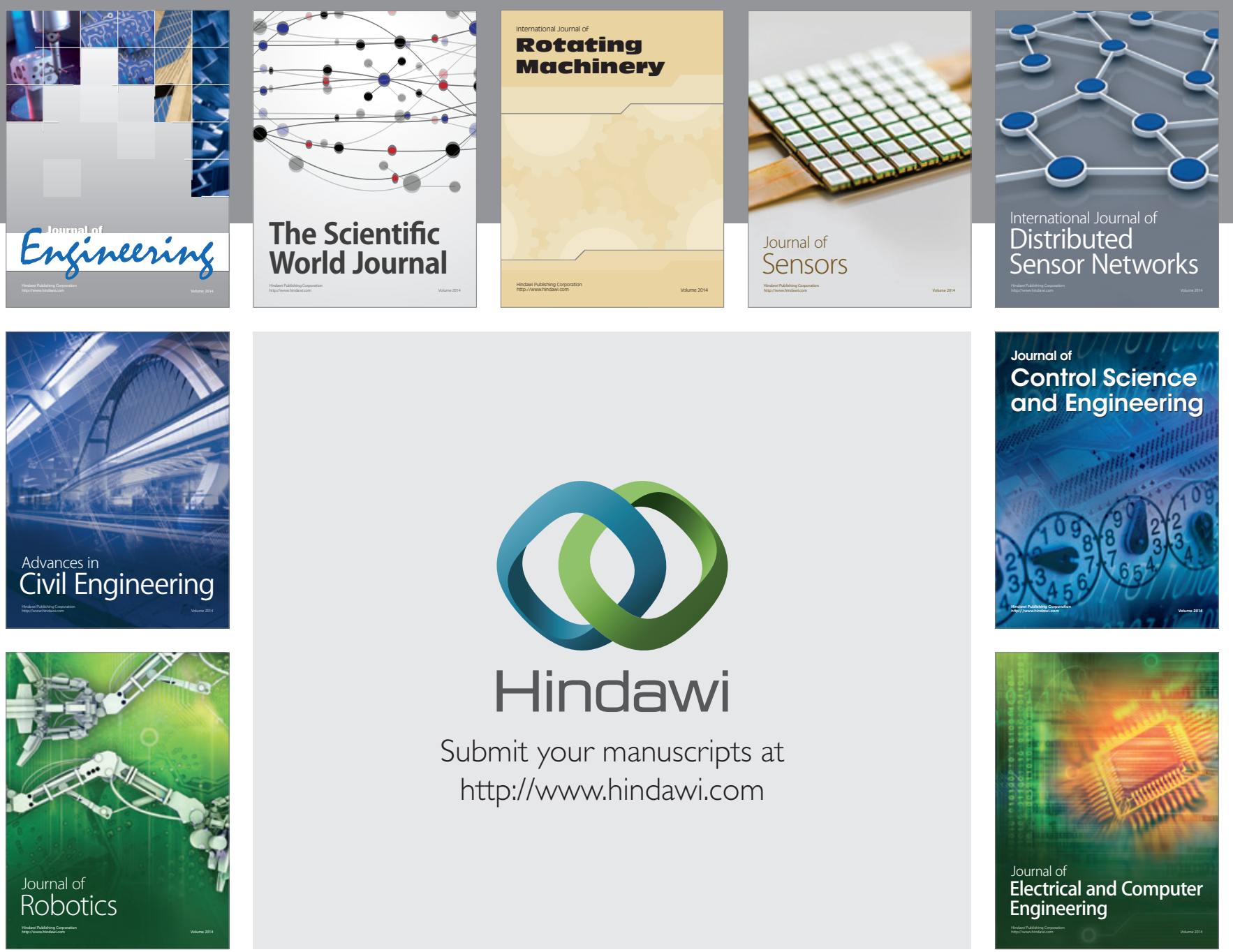

Submit your manuscripts at

http://www.hindawi.com
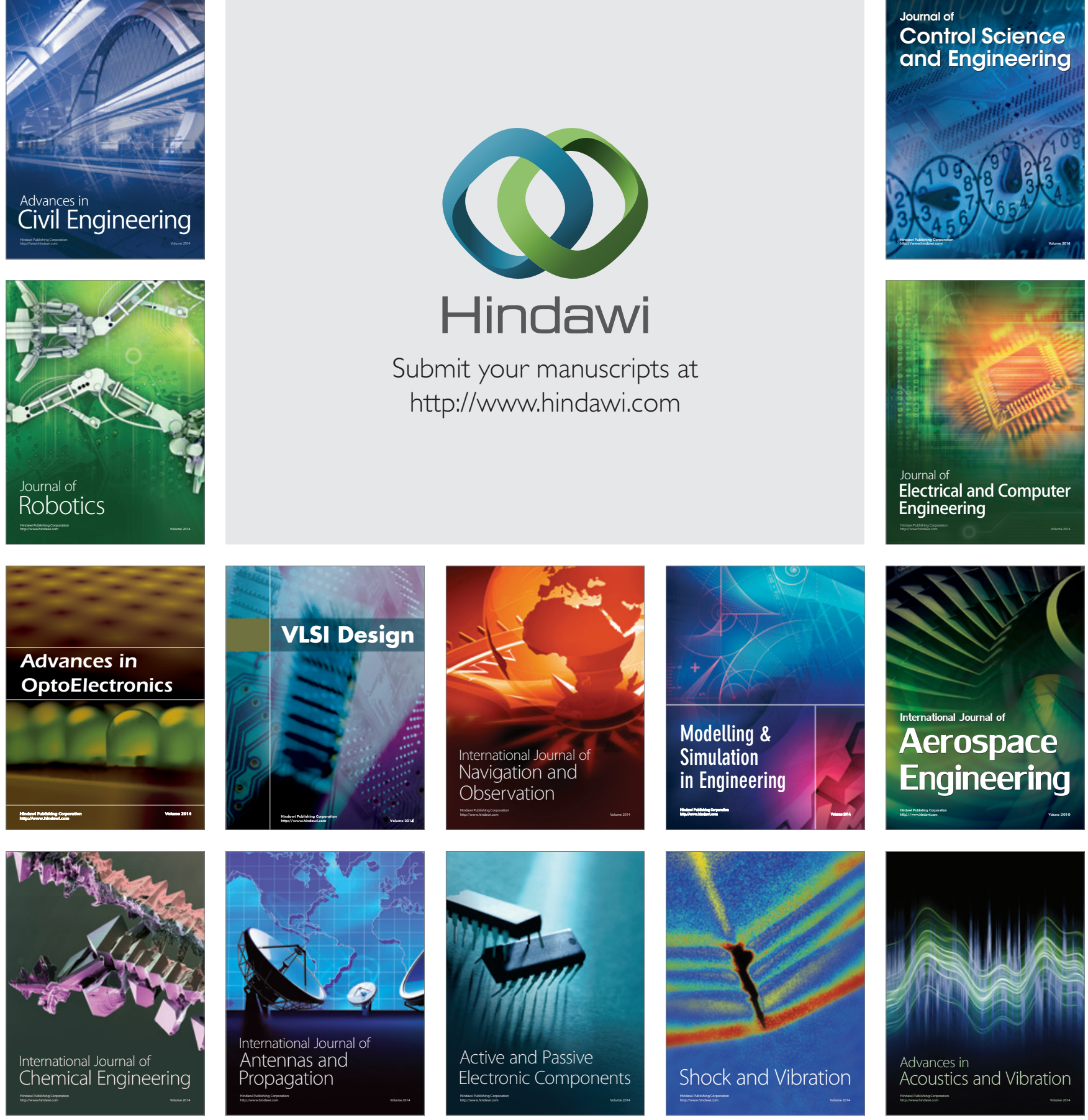